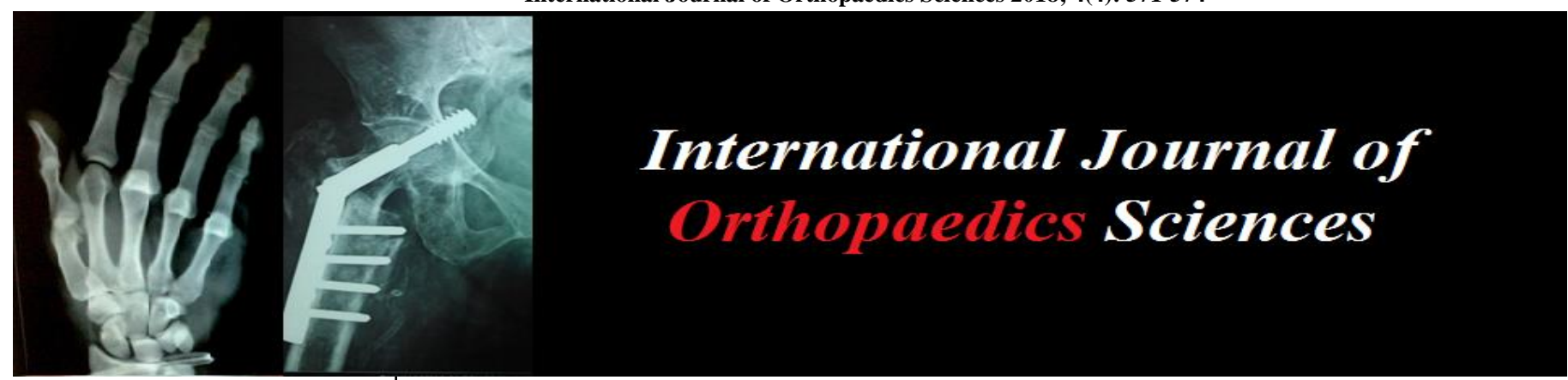

ISSN: $2395-1958$

IJOS 2018; 4(4): 571-574

(C) 2018 IJOS

www.orthopaper.com

Received: 04-08-2018

Accepted: 08-09-2018

Dr. Khaja Adil Ahmed

Assistant Professor, department of Orthopaedics, Bhaskar medical college, Yenkapally, Moinabad, Ranga Ruddy,

Telangana, India

Dr. Mir Zia Ur Rahman Ali Department of Orthopaedics, Bhaskar Medical College, Yenkapally, Moinabad, Ranga Reddy, Telangana, India

\section{Comparatively increased complications in obese patients undergoing total hip arthroplasty through a direct anterior approach}

\author{
Dr. Khaja Adil Ahmed and Dr. Mir Zia Ur Rahman Ali
}

DOI: https://doi.org/10.22271/ortho.2018.v4.i4g.62

\section{Abstract}

Background: Direct Anterior IP replacement is a minimally invasive surgical technique. This approach involves a 3 to 4 inch incision on the front of the hip that allows the joint to be replaced by moving muscles aside along their natural tissue planes, without detaching any tendons. This approach often results in quicker recovery, less pain, and more normal function after hip replacement. The Direct Anterior (DA) approach for Total Hip Arthroplasty (THA) has demonstrated successful short term outcomes in several studies. However, there is no consensus about which patients are appropriate candidates for DA Total Hip Arthroplasty. It is also unclear if short term outcomes in obese patients undergoing THA through a DA approach are elevated in comparison to non-obese patients.

Objective: The aim of this study was to evaluate complication rates and short term outcomes of obese, pre-obese, and normal Body Mass Index (BMI) patients undergoing Total Hip Arthroplasty with a Direct Anterior approach in a consecutive group of patients.

Design: A retrospective study of 200 consecutive patients who underwent unilateral THA through a DA approach for osteoarthritis or avascular necrosis during the early peri-operative period.

Duration: November 2016 to December 2017.

Setting: Department of Orthopaedics, Bhaskar Medical College, Hyderabad.

Participants: 200 consecutive patients who underwent unilateral THA through a DA approach.

Methods: All adult patients undergoing primary total hip arthroplasty were eligible for inclusion. A retrospective chart review of 210 consecutive patients who underwent unilateral primary THA through a DA approach was conducted. All 200 cases used the same implants. The direct anterior approach utilized a modified Smith-Peterson interval. A standard operating table was used along with a femoral elevator. Routine use of fluoroscopy was not used. Unless contraindicated, patients were treated with a standardized pain protocol. A local tissue pain injection was administered as well as intravenous tranexamic acid. The same closure technique was utilized for all patients. Physical therapy followed a standardized protocol and began on postoperative day 0. Standard post-discharge follow-up was at two and six weeks. All patients were placed on aspirin $325 \mathrm{mg}$ twice daily for DVT prophylaxis. Electronic and paper medical records were reviewed to determine surgical time, length of stay, discharge disposition, major and minor complications, and short-term outcome measures. Major and minor complications were separately described.

Results: The obese group demonstrated increased surgical times, as well as increased length of stay, narcotic use, and assistive device use at two weeks. In addition, major complications and wound complications were both significantly increased in the obese group. There was also a trend toward increased use of rehabilitation facilities in the obese group at disposition.

Conclusion: Obese patients undergoing a DA approach have many times increase in major and wound complications compared to patients with a BMI <30. Obese patients also demonstrated significant increases in operative time, use of narcotics, use of assistive devices, and length of stay.

Keywords: total hip arthroplasty, direct anterior approach, obese, BMI

\section{Introduction}

Total hip arthroplasty is an orthopedic procedure that involves the surgical excision of the head and proximal neck of the femur and removal of the acetabular cartilage and subchondral bone. An artificial canal is created in the proximal medullary region of the femur, and a metal femoral prosthesis, composed of a stem and small-diameter head, is inserted into the femoral medullary canal. An acetabular component composed of a high-molecular-weight polyethylene

\section{Dr. Mir Zia Ur Rahman Al} Department of Orthopaedics, Bhaskar Medical College, Yenkapally, Moinabad, Ranga Reddy, Telangana, India 
articulating surface is inserted proximally into the enlarged acetabular space. To yield successful results, these total hip arthroplasty components must be fixed firmly to the bone, either with polymethylmethacrylate cement or, in more recent uncemented designs, by bony ingrowth into a porous coating on the implant, resulting in "biologic" fixation. Total hip arthroplasty (THA) has become one of the most reliable and patient-requested surgical interventions in all medicine. The procedure can be performed using a variety of surgical approaches, but the posterior approach, direct lateral approach, and direct anterior approach are by far the most common across the globe. Direct anterior hip replacement is a minimally invasive surgical technique. This approach involves a 3 to 4 inch incision on the front of the hip that allows the joint to be replaced by moving muscles aside along their natural tissue planes, without detaching any tendons. This approach often results in quicker recovery, less pain, and more normal function after hip replacement. Because the tendons aren't detached from the hip during direct anterior hip replacement, hip precautions are typically not necessary. This allows patients to return to normal daily activities shortly after surgery with a reduced risk of dislocation. The Direct Anterior (DA) approach for Total Hip Arthroplasty (THA) has demonstrated successful short term outcomes in several studies. However, there is no consensus about which patients are appropriate candidates for DA Total Hip Arthroplasty. It is also unclear if short term outcomes in obese patients undergoing THA through a DA approach are elevated in comparison to non-obese patients. The aim of this study was to evaluate complication rates and short term outcomes of obese, pre-obese, and normal Body Mass Index (BMI) patients undergoing Total Hip Arthroplasty with a Direct Anterior approach in a consecutive group of patients.

\section{Materials and Methods}

This is a retrospective study of 200 consecutive patients who underwent unilateral THA through a DA approach for osteoarthritis or avascular necrosis during the early perioperative period. All adult patients undergoing primary total hip arthroplasty were eligible for inclusion. A retrospective chart review of 210 consecutive patients who underwent unilateral primary THA through a DA approach was conducted. All 200 cases used the same implants. The direct anterior approach utilized a modified Smith-Peterson interval. A standard operating table was used along with a femoral elevator. Routine use of fluoroscopy was not used. Unless contraindicated, patients were treated with a standardized pain protocol. A local tissue pain injection was administered as well as intravenous tranexamic acid. The same closure technique was utilized for all patients, Derma bond was applied topically and an occlusive foam dressing was applied.
Physical therapy followed a standardized protocol and began on postoperative day 0 . Standard post-discharge follow-up was at two and six weeks. All patients were placed on aspirin $325 \mathrm{mg}$ twice daily for DVT prophylaxis. Electronic and paper medical records were reviewed to determine surgical time, length of stay, discharge disposition, major and minor complications, and short-term outcome measures. Major and minor complications were separately described.

\section{Observations and Results}

Patient Characteristics by Bmi and Age

Table 1: Patient Characteristics by BMI and Age

\begin{tabular}{|c|c|c|}
\hline BMI Group & Mean & Standard Deviation \\
\hline \multicolumn{3}{|c|}{ Normal(n=25) } \\
\hline BMI & 21.34 & 1.334 \\
\hline Age & 59.98 & 11.006 \\
\hline \multicolumn{3}{|c|}{ Pre-Obese(n=75) } \\
\hline BMI & 26.98 & 1.241 \\
\hline Age & 66.12 & 11.112 \\
\hline \multicolumn{3}{|c|}{ Obese Class I(n=50) } \\
\hline BMI & 30.98 & 1.670 \\
\hline Age & 58.67 & 10.665 \\
\hline \multicolumn{3}{|c|}{ Obese Class II(35) } \\
\hline BMI & 38.76 & 1.714 \\
\hline Age & 56.13 & 8.998 \\
\hline \multicolumn{3}{|c|}{ Obese Class III(15) } \\
\hline BMI & 43.99 & 3.665 \\
\hline Age & 52.90 & 14.997 \\
\hline
\end{tabular}

Two hundred consecutive patients, undergoing total hip arthroplasty through a direct anterior approach by a single experienced surgeon, were evaluated (Table 1). Comparing normal and pre-obese patients $(\mathrm{BMI}<30)$ to a combined group of WHO class I, II, and III obese patients, wound complication rates were $4.0 \%$ in the $\mathrm{BMI}>30$ group, and $9 \%$ in the combined group.

Odds Ratio Estimates: Normal and Pre-Obese (Combined) Vs Obese

Table 2: Odds Ratio Estimates: Normal and Pre-Obese (Combined) vs Obese

\begin{tabular}{|c|c|c|}
\hline & Odds Ratio & P Value \\
\hline Minor Complications & 3.788 & 0.0419 \\
\hline Major Complications & 8.431 & 0.0488 \\
\hline
\end{tabular}

The odds ratio showed a 7.6 fold increase for major complications and a 2.8 fold increase for minor complications in the obese group when compared to the normal and preobese group combined.

Comparison of Normal and Pre-Obese (Combined) Vs Obese

Table 3: Comparison of Normal and Pre-Obese (Combined) vs Obese

\begin{tabular}{|c|c|c|}
\hline Characteristics & Normal and Pre-Obese(N=100) & Obese(N=100) \\
\hline \multicolumn{3}{|c|}{ Minor Complications } \\
\hline Yes & $6(6 \%)$ & $12(12 \%)$ \\
\hline No & $94(94 \%)$ & $88(88 \%)$ \\
\hline Yes & Major Complications \\
\hline No & $2(2 \%)$ & $7(7 \%)$ \\
\hline ASA Score & $98(98 \%)$ & $93(93 \%)$ \\
\hline Pain-Pre & $2.3 \pm 0.4$ & $2.6 \pm 0.5$ \\
\hline Pain-2 Weeks & $6.7 \pm 1.9$ & $7.8 \pm 2.2$ \\
\hline Pain-6 Weeks & $2.2 \pm 2.0$ & $2.6 \pm 2.4$ \\
\hline \multicolumn{2}{|c|}{$1.5 \pm 2.2$} & $2.0 \pm 2.3$ \\
\hline
\end{tabular}




\begin{tabular}{|c|c|c|}
\hline Yes & $31(31 \%)$ & $42(42 \%)$ \\
\hline No & $69(69 \%)$ & $58(58 \%)$ \\
\hline \multicolumn{3}{|c|}{ Narcotics(6 Weeks) } \\
\hline Yes & $2(2 \%)$ & $8(8 \%)$ \\
\hline No & $98(98 \%)$ & $92(92 \%)$ \\
\hline Yes & Assistive Device(2 Weeks) & $84(84 \%)$ \\
\hline No & $79(79 \%)$ & $16(16 \%)$ \\
\hline \multicolumn{3}{|c|}{$21(21 \%)$} \\
\hline Yes & Assistive Device(6 Weeks) & $34(34 \%)$ \\
\hline No & $16(16 \%)$ & $66(66 \%)$ \\
\hline Length Of Stay & $84(84 \%)$ & $2.7 \pm 1.0$ \\
\hline \multicolumn{2}{|c|}{$2.4 \pm 1.1$} \\
\hline Home & Discharge Disposition & $81(81 \%)$ \\
\hline Rehabilitation & $91(91 \%)$ & $19(19 \%)$ \\
\hline
\end{tabular}

There were a total of nine major complications (Table 3). Seven major complications occurred in the obese group, with three requiring a return to the operating room. Three patients required a two-stage revision procedure for infection, one required stem revision for aseptic loosening, and one with intra-operative medial femoral perforation and greater trochanteric fracture. Two patients in the obese group were diagnosed with a pulmonary embolism and treated with anticoagulation. The two major complications in the preobese group required a return to the operating room for infection.

\section{Discussion}

The direct anterior approach utilizing a muscle-sparing technique has gained popularity in recent years for primary THA due its potential for improved post-operative pain, recovery and strengthening. Few studies, however, have been performed analyzing if these benefits remain significant in the obese population. Obese patients present a significant challenge for THA surgeons due to their large body habitus. We have noted that in many obese patients there is a relatively small amount of subcutaneous adipose tissue along the anterior aspect of the proximal thigh where the incision and dissection is located for the DA approach which can actually allow for a relatively routine operative procedure. Even in large patients it is often possible to mobilize and reposition the abdominal pannus away from the incision and operative field allowing for an easier exposure. Despite the many potential benefits of the direct anterior approach, there remains a real concern regarding total complication rates and the learning curve associated with its use in routine practice. Despite the many potential benefits of the direct anterior approach, there remains a real concern regarding total complication rates and the learning curve associated with its use in routine practice. This study reveals a significant increase in complication rates for obese patients in comparison to non-obese patients using the DA approach. In a prospective matched cohort study using an anterolateral approach Chee et al. reported a $9 \%$ major complication rate and a $13 \%$ minor wound complication rate for their obese patients which had an average BMI of 37.9. In comparison, our complication rates were $6.3 \%$ for major, and $10 \%$ for minor wound complications. Michalka et al. found a $14 \%$ major complication rate and a $12.3 \%$ minor complication rate in their study of obese patients undergoing THA using a posterior approach. A study by Dowsey and Choong of obese patients using a posterior approach reported a $4.4 \%$ acute infection rate in comparison to our rate of $2.5 \%$. Overall our experience demonstrates an increased complication rate for the DA approach in obese patients that is comparable to the published data for other operative approaches, which does not appear to be directly attributable to the DA approach itself. Limitations of this study include a retrospective design and relatively short-term follow-up with no specific outcomes instruments; however, the focus of the study was designed to analyze early complications in a specific obese population rather than long-term functional outcomes. In addition, preoperative ASA scores were also found to be higher among the obese group indicating higher comorbidities which may contribute to wound healing problems, length of stay, and use of rehabilitation facilities. This study only provides data as to whether wound complications may be an independent risk factor for the DA approach by comparing our complication rates to the published complication rates for other approaches available in the literature. This study demonstrates that the complication rate for obese patients undergoing THA utilizing a DA approach is significantly increased in comparison to normal and pre-obese patients.

\section{Conclusions}

Obese patients undergoing a DA approach have many times increase in major and wound complications compared to patients with a BMI <30. Obese patients also demonstrated significant increases in operative time, use of narcotics, use of assistive devices, and length of stay.

\section{References}

1. Felson DT, Lawrence RC, Dieppe PA, Hirsch R, Helmick CG, Jordan JM, et al. Osteoarthritis: New insights. Part 1: The disease and its risk factors. Ann Intern Med. 2000; 133:635-46.

2. Guccione AA, Felson DT, Anderson JJ, Anthony JM, Zhang Y, Wilson PW, et al. The effects of specific medical conditions on the functional limitations of elders in the Framingham Study. Am J Public Health. 1994; 84:351-8.

3. Jordan JM, Helmick CG, Renner JB, Luta G, Dragomir AD, Woodard J, et al. Prevalence of hip symptoms and radiographic and symptomatic hip osteoarthritis in African Americans and Caucasians: The Johnston County Osteoarthritis Project. J Rheumatol. 2009; 36:809-15.

4. Lawrence RC, Felson DT, Helmick CG, Arnold LM, Choi H, Deyo RA, et al. Estimates of the prevalence of arthritis and other rheumatic conditions in the United States. Part II. Arthritis Rheum. 2008; 58:26-35.

5. Hootman JM, Helmick CG. Projections of US prevalence of arthritis and associated activity limitations. Arthritis Rheum. 2006; 54:226-9.

6. Morrey BF. A historical perspective of hip arthroplasty and reconstructive surgery. In: Cashman J, Goyal N, 
Parvizi J, editors. The Hip: Preservation, Replacement and Revision. Brooklandville, MD: Data Trace Publishing Company. 2015; 1(1):19.

7. Charnley J. Total hip replacement by low-friction arthroplasty. Clin Orthop Relat Res. 1970; 72:7-21.

8. Maradit Kremers H, Larson DR, Crowson CS, Kremers WK, Washington RE, Steiner CA, et al. Prevalence of total hip and knee replacement in the United States. J Bone Joint Surg Am. 2015; 97:1386-97.

9. Kremers MH, Visscher SL, Kremers WK, et al. Obesity increases length of stay and direct medical costs in total hip arthroplasty. Clin Orthop Relat Res. 2014; 472(4): 1232.

10. Elson LC, Barr CJ, Chandran SE, et al. Are morbidly obese patients undergoing total hip arthroplasty at an increased risk for component malpositioning? J Arthroplasty. 2013; 28(8):41.

11. Pugely AJ, Callaghan JJ, Martin CT, et al. Incidence of and risk factors for 30-day readmission following elective primary total joint arthroplasty: analysis from the ACSNSQIP. J Arthroplasty 2013; 28(9):1499.

12. Bozic KJ, Ward DT, Lau EC, et al. Risk factors for periprosthetic joint infection following primary total hip arthroplasty: a case control study. J Arthroplasty. 2014; 29(1):154.

13. Jewett BA, Collis DK. High complication rate with anterior total hip arthroplasties on a fracture table. Clin Orthop Relat Res 2011; 469:503.

14. Bhandari M, Matta JM, Dodgin D, et al. Outcomes following the single-incision anterior approach to total hip arthroplasty: a multicenter observational study. Orthop Clin North Am. 2009; 40:329.

15. Christensen CP, Karthikeyan T, Jacobs CA. Greater prevalence of wound complications requiring reoperation with direct anterior approach total hip arthroplasty. J Arthroplasty. 2014; 29(9):1839.

16. Cook SD, McCluskey LC, Martin PC, Haddad RJ, Jr Inflammatory response in retrieved non-cemented porous-coated implants. Clin Orthop Relat Res. 1991; (264):209-222.

17. Haddad RJ, Jr Cook SD, Thomas KA. Biological fixation of porous-coated implants. J Bone Joint Surg Am. 1987; 69(9):1459-1466.

18. Collier JP, Mayor MB, Chae JC, Surprenant VA, Surprenant HP, Dauphinais LA. Macroscopic and microscopic evidence of prosthetic fixation with porouscoated materials. Clin Orthop Relat Res. 1988; (235):173-180.

19. Harris WH, Maloney WJ. Hybrid total hip arthroplasty. Clin Orthop Relat Res. 1989; (249):21-29.

20. Weber ER, Daube JR, Coventry MB. Peripheral neuropathies associated with total hip arthroplasty. J Bone Joint Surg Am. 1976; 58(1):66-69.

21. Haake DA, Berkman SA. Venous thromboembolic disease after hip surgery. Risk factors, prophylaxis, and diagnosis. Clin Orthop Relat Res. 1989; (242):212-231. 